\title{
POLINIZACIÓN CONTROLADA EN UNGURAHUI (Oenocarpus bataua Mart.) TAMBOPATA- MADRE DE DIOS
}

\section{Telésforo VÁSQUEZ ZAVALETA ${ }^{1}$, Ives QUISPE GOMES ${ }^{2}$}

1 Instituto de Investigaciones de la Amazonía Peruana IIAP - Pto. Maldonado, J irón Ica 1162 Puerto M aldonado, Madre de Dios. Perú, email: tvasquezz64@ yahoo.es Telefax. 082571897

2 Universidad Nacional Amazónica de Madre de Dios. Av. Dos de Mayo N960.Puerto Maldonado-Madre de Dios-Perú. Telefax. 082573186

\section{RESUMEN}

El propósito del estudio de polinización controlada en ungurahui (Oenocarpus bataua Mart.), fue determinar la posibilidad de poder incrementar la producción de frutos en los racimos de esta especie; para tal efecto se ensayaron cuatro tratamientos, en tres racimos de igual número de árboles diferentes y distantes; los resultados indican que existió significancia entre el tratamiento T1 (Raquillas sin flores masculinas, con polinización manual, aisladas con bolsas de polietileno) frente al tratamiento, T3 (Raquillas con flor masculina, con polinización cruzada, sin aislar (Testigo) (polinización abierta)), mas no así con el T2 (Raquillas con flor masculina, sin polinizar y sin aislar). La producción lograda con polinización controlada fue en promedio de $32 \%$ frente a $17 \%$ que se obtuvo con polinización libre.

PALABRAS CLAVES: Madre de Dios, Ungurahui, Oenocarpus bataua, polinización.

\section{CONTROLLED POLLINATION IN UNGURAHUI (Oenocarpus bataua Mart.) TAM BOPATA-M ADRE DE DIOS}

\section{ABSTRACT}

The purpose of controlled pollination in ungurahui (Oenocarpus bataua Mart.), was to determine the possibility to increase production of fruits in clusters of this species for this purpose four treatments were tested in three clusters of equal number of different and distant trees; the results indicate there was significance between treatment T1 (pollinated flowers, insulated bag) compared to treatment T3 (rachilla with male flowers without pollination and uninsulated (Control) (open pollinated)) but not so with the T2 (rachilla with male flowers, pollinated, nonisolated). The production achieved with controlled pollination was on average $32 \%$ versus $17 \%$ that obtained with open-pollinated.

KEYW ORDS: Madre de Dios, Ungurahui, Oenocarpus bataua, pollination. 


\section{INTRODUCCIÓN}

Los trabajaros realizados sobre Oenocarpus bataua, en los últimos años, tratan sobre su distribución geográfica, características botánicas, aspectos taxonómicos (Henderson et al., 1995), usos y alternativas de aprovechamiento (Balick, 1986; Mejía, 1992, 1988), aspectos muy generales sobre ecología y biología reproductiva (Balick, 1986), fenología (Collazos \& Mejía, 1988; Ruiz \& Alentar, 2004), polinización (García, 1988) y algunos aspectos demográficos (Henderson et al., 1995)

Ensayos de polinizaciones controladas realizados por Nuñez \& Rojas (2008), indican que O. bataua es predominantemente xenógama, debido a que la proporción de semillas viables formadas por alogamia fue de $71 \%$ sin diferencias significativas con las pruebas de polinización abierta $92 \%$. No se presenta apomixis y la autopolinización es improbable. El bajo porcentaje en la viabilidad de sus semillas y el bajo valor del ISI (0.019) dan prueba de su autoincompatibilidad.

Los aromas de las flores del O. bataua están constituidos por compuestos de los cuales el $\alpha$ gurjuneno constituye el $43 \%$, el ciclozativeno el $25 \%$, $\mathrm{y}$ el trans-cariofileno el $9,8 \%$; son las sustancias predominantes en la mezcla. (Núñez \& Rojas, 2008)

El mismo autor, estimó la cantidad promedio de granos de polen de $O$. bataua en $12946 \pm 3245$ granos por antera, $129464 \pm 6721$ por flor, y $9440514880 \pm$ 56784 porinflorescencia.

En la fase masculina de la floración de O. bataua, ( Op.cit), reporto visitas de 81 especies de insectos y otros artrópodos: Curculionidae con 22 especies, Scarabaeidae con 3 especies, Staphylinidae con 5 especies y Nitidulidae con 2 especies. Las especies de abejas sociales (Meliponinae) y los parasitoides ambos del orden Hymenoptera, presentaron 9 y 14 especies respectivamente. La diversidad de visitantes en la fase femenina fue menor con 32 especies de insectos visitando las flores cuando los estigmas aún permanecían receptivos.

En O. bataua, el fenómeno de protandria, ántesis pistilada, se inicia una vez concluida la ántesis estaminada haciendo suponer que la polinización es íntegramente cruzada (Xenogamia) y que únicamente un $15 \%$ de flores pistiladas llegan al final como frutos maduros, debido, entre otros factores, a la posible deficiencia de polinización de los mismos. (Vásquez, 2007)

El propósito fue determinar si con polinización controlada se podría incrementar la productividad de frutos de ungurahui $O$. bataua Mart, para mejorar producción en futuras plantaciones o para hacer mejoramiento genético con la especie.

\section{ATERIALES Y MÉTODOS}

\section{OBTENCIÓN Y PREPARACIÓN DEL POLEN.}

El polen de Ungurahui (Oenocarpus bataua Mart.) fue colectado de tres individuos que se encontraban en floración, en el área del Centro Experimental Fiztcarrald - IIAP - MDD ubicado a $21 \mathrm{~km}$ de la carretera Pto. Maldonado - Cusco. Los individuos escogidos se encuentran ubicados en las coordenadas UTM siguientes: árbol Nº 8 en 463635E - 8601240N; árbol $\mathrm{N}^{\circ} 23$ en $463580 \mathrm{E}-8601461 \mathrm{~N}$ y árbol $\mathrm{N}^{\circ} 38$ en 463614-8601514N

Para obtener el polen se usaron bolsas de papel kraft que se usaron como trampas, estas, fueron colocadas por las tardes y recogidas por las mañanas del día siguiente; la colecta consistió en embolsar un grupo de raquillas cuyas flores estaminadas estaban en antesis durante 12 a 15 horas. Retiradas las bolsas, que estaban húmedas, se les seco en estufa por 6 horas a 40 ${ }^{\circ} \mathrm{C}$; Luego se tamizo en tamiz de malla 100. Para la conservación del polen se colocó en frascos sin taparlos y se coloco dentro de un desecador por 24 horas más, esto con la finalidad de reducir la humedad y así el almacenado sea más exitoso. Luego se guardan bajo refrigeración a una temperatura de aprox. $4{ }^{\circ} \mathrm{C}$.

\section{EVALUACIÓN DE VIABILIDADDEL POLEN}

La viabilidad se determino mediante la prueba de germinación del polen, habiéndose utilizado una solución azucarada al 10\% (azúcar blanca de mesa)

En la solución, puesta en placas petri, previamente esterilizada, se realizó la siembra del polen; para el cual se hizo uso de un pincel, previamente impregnado con granos de polen. Se realizaron tres repeticiones por prueba y se ponían a incubación por cuatro horas. La incubación se efectuó en oscuridad, a $29^{\circ} \mathrm{C}$ y $100 \%$ de humedad relativa (cámara húmeda). Pasado las cuatro horas después de la siembra del polen se procedía a la observación al microscopio (x40). Por cada placa se observaron 500 granos de polen entre germinados y no germinados. Se consideró que un grano de polen había germinado cuando la longitud del tubo polínico fue igual o superior al diámetro del grano de polen.

Esta prueba es importante porque nos aseguraba que el polen a utilizar mantiene viabilidad, considerando el $50 \%$ de viabilidad como aceptable.

\section{POLINIZACIÓN CONTROLADA}

\section{IDENTIFICACIÓN DEÁRBOLES Y DISEÑO EXPERIM ENTAL}

Fueron seleccionados tres árboles de ungurahui en floración en la estación experimental Fitzcarrald. 
El diseño empleado fue bloques completamente al azar; cada árbol represento a un bloque; para cada tratamiento se hizo tres repeticiones, representados por el promedio porcentual de flores fecundadas de 7 raquillas.

En los tratamientos T1 y T4, antes que se inicie la antesis estaminada se retiraron las flores estaminadas y se aislaron con bolsas transparentes de polietileno

\section{TRATAM IENTOS}

Los tratamientos evaluados fueron:

- $\mathrm{T} 1=$ Raquillas sin flores masculinas, con polinización manual, aisladas con bolsas de polietileno,

- $\mathrm{T} 2=$ Raquillas con flores masculinas, con polinización manual, sin aislar,

- $\mathrm{T} 3=$ Raquillas con flor masculina, con polinización cruzada, sin aislar (Testigo)

- T4= Raquillas sin flor masculina, sin polinizar, aisladas con bolsa de polietileno.

\section{POLINIZACIÓN Y MONITOREO}

Los tratamientos $\mathrm{T} 1$ y T2 se hicieron con polen de otro árbol, previa evaluación de viabilidad superior al $50 \%$. Con la ayuda de un pincel de cerdas impregnada de polen se tocaba la flor pistilada en antesis, esta operación se realizo todas las mañanas hasta que todas las flores pistiladas hayan entrado en antesis. El tratamiento T3 fue libre, en tanto T4, desde el inicio de la apertura de la inflorescencia, se le retiraron las flores masculinas y se le aisló con balsa de polipropileno transparente.

El monitoreo se ejecuto, cada 60 días hasta la cosecha de los frutos que fue a lo 300 días de iniciada la polinización.

\section{RESULTADOS Y DISCUSIÓN}

Después de los 60 días de realizada la polinización se observó una drástica pérdida de flores pistiladas, siendo de hasta un $82 \%$ para el T4, mientras la que menos perdió fue $\mathrm{T} 1$ de un $50 \%$; quedando aquellas que probablemente han sido fertilizadas. Sin embargo, a los 180 días, la cantidad de flores fertilizadas continuaron disminuyendo. Al momento de la cosecha, que fue a los 300 días después de realizada la polinización, los resultados fueron de la siguiente manera: $32 \%$ para $\mathrm{T} 1,26 \%$ para $\mathrm{T} 2,17 \%$ para $\mathrm{T} 3$ y $0 \%$ para T4 (Figura 1). Los resultados de polinización libre coincidió con las evaluaciones realizadas por Vásquez (2007), para racimos de polinización libre, que fue del $15 \%$; pero con la polinización controlada se logró $32 \%$ lo que indica que la producción se duplico; en tanto experiencias con otras especies como el reportado por Freitas (2004), para la especie aguaje (Mauritia flexuosa) triplico la producción con polinización controlada a polinización libre $(32.43 \%$ vs $11.33 \%$ respectivamente), asimismo, Cavalcante (2008), reporta para castaña (Bertholletia excelsa) un incremento seis veces más con polinización controlada que con polinización libre $(19.33 \%$ vs $3.05 \%$ respectivamente). Por tanto, mejorar la productividad en el ungurahui y otras especies es factible mediante polinización controlada (manual).

Del análisis de varianza, Tabla 1, se desprende que existe alta significancia entre los tratamientos; mediante la prueba de Tukey se determinó que el tratamientos $\mathrm{T} 1$ es significativo con $\mathrm{T} 3$ y muy significativo con $\mathrm{T} 4$, es decir el tratamiento con polinización controlada y aislada fue positiva frente a polinización libre (normal); el resultado con T4, fue lo esperado por que estas flores, femeninas, fueron aisladas totalmente (Ver Tabla 2).

El hecho que T3 no difiere significativamente con T2 pero si con $\mathrm{T} 1$, nos indica que probablemente la polinización controlada (manual) no tendría importancia en el incremento de la productividad si es que no se realiza adicionalmente tratamientos de sanidad o aislamientos de los racimos, tratamiento adicional que evitaría ataques de enfermedades o insectos, estas serían las razones que generan la diferencia significativa entre T1 y T3. 
FO LIA

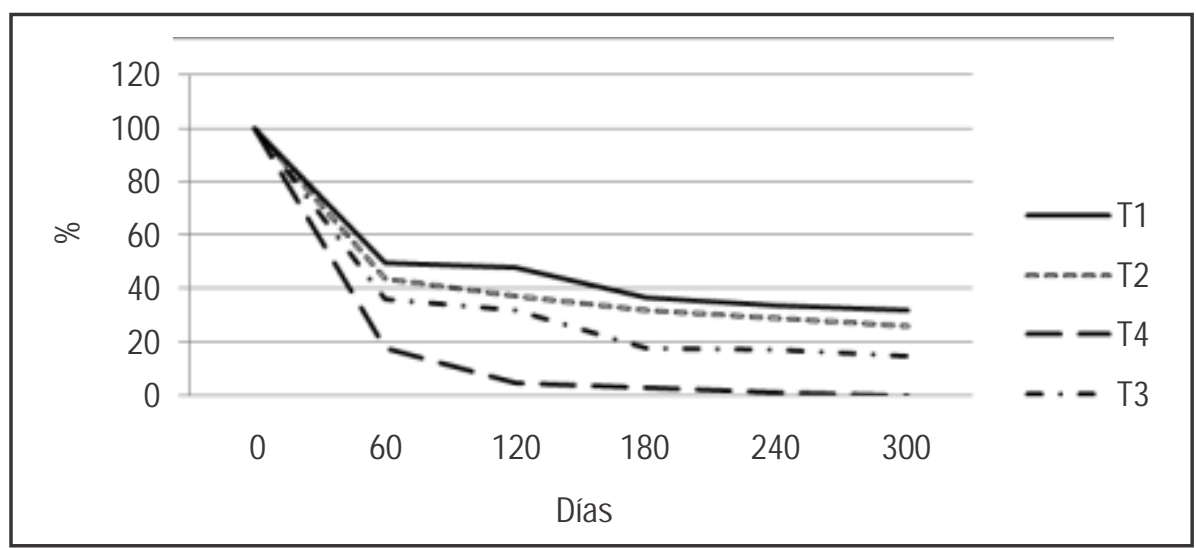

Figura 1. Evolución del porcentaje de flores fertilizadas después de la polinización de Ungurahui Oenocarpus bataua controlada en 300 días de tratamiento.

Tabla 1. Análisis de varianza de polinización controlada en ungurahui Oenocarpus bataua.

\begin{tabular}{lrrrr}
\hline \multicolumn{1}{c}{ FV } & GL & SQ & QM & FC \\
\hline Tratamientos & 3 & 1693.67 & 564.55 & 21.74 \\
Bloques & 2 & 186.17 & 93.08 & $* *$ \\
Error experimental & 6 & 155.83 & 25.97 & \\
ToTal & 11 & 2035.67 & & \\
\hline Ft al 5\% & 4.76 & $(\mathrm{Gl} 3,6)$ & $\mathrm{CV} \% \mathrm{~T} 1=28.001$ & \\
al 1\% & 9.78 & & CV\% T2 $=10.25$ & \\
CV\% T3 & 29.3 & & &
\end{tabular}

Tabla 2. Comparación de medias por tratamiento en polinización ungurahui Oenocarpus bataua.

\begin{tabular}{cccc}
\hline \multirow{2}{*}{ TRATAMIENTOS } & \multirow{2}{*}{ MEDIA } & \multicolumn{2}{c}{ SIGNIFICANCIA } \\
\cline { 3 - 4 } & & $\mathbf{0 . 0 5 \%}$ & $\mathbf{0 . 0 1 \%}$ \\
\hline T1 & 32 & $\mathrm{a}$ & $\mathrm{A}$ \\
$\mathrm{T} 2$ & 26 & $\mathrm{ab}$ & $\mathrm{A}$ \\
$\mathrm{T} 3$ & 17 & $\mathrm{~b}$ & $\mathrm{AB}$ \\
$\mathrm{T} 4$ & 0 & $\mathrm{c}$ & $\mathrm{B}$ \\
\hline
\end{tabular}

Valores de tukey a $5 \%=14.56$ y a $1 \%=20.89$ 


\section{CONCLUSIONES}

La polinización controlada es un una buena alternativa para incrementar la productividad de ungurahui; sin embargo, esto no sería factible lograrlo si es que no se practica un aislamiento o alguna forma de sanidad a los racimos.

\section{BIBLIOGRAFÍA CITADA}

Balick, M. J. 1986. Systematics and Economic Botany of the Oenocarpus-Jessenia (Palmae) Complex. in Advances in Economic Botany, 3: 1-140.

Cavalcante, M. C. 2008. Visitantes florais e polinização da castanha-do-brasil (Bertholletia excelsa) em cultivo na amazônia central.Tesis de grado de Maestria de Zootecnica, Universidad Federal do Ceará centro de Ciencias Agrarias Departamento de Zootecnia. Fortaleza-CE. Brasil $77 \mathrm{pp}$.

Collazos, M.; M. Mejía. 1988. Fenología y poscosecha de milpesos Jessenia bataua (Mart) Burret. Acta Agronómica, 38:53-63.

Freitas, L. 2004. Domesticación y Servicios Ambientales del Aguaje Mauritia flexuosa L.f. en 1 a A m a z o ní a P e r u a $\mathrm{n}$ a. http://www.iiap.org.pe/Upload/Conferencia/CON F5.ppt. Acceso: 30/10/2010
García, S. M. 1988. Observaciones de polinización en Jessenia bataua (Arecaceae). - tesis de licenciatura, Pontificia Universidad Católica del Ecuador. Departamento de Ciencias Biológicas, Quito, Ecuador 69pp.

Henderson, A; Galeano, G; Bernal, R. 1995. Palms of the Americas Princenton University Pres; New Jersey 08340, 1995

Mejía, K. 1992. Las Palmeras en los Mercados de Iquitos. Bull. Inst. Fr. d'études Andines, 21(2): 755-769.

Mejía, K.1988. Utilization of palms in eleven mestizo villages of the Peruvians Amazon (Ucayali River, Department of Loreto).-Adv. Economía Botánica 6:130-136

Nuñez, L.; Roja, R. 2008. Biología reproductiva y ecología de la polinización de la palma milpesos Oenocarpus bataua en los andes colombianos. Caldasia, 30(1):101-125. 2008.

Ruiz, R. R.; Alencar, J. C. 2004. Comportamiento fenológico da palmeira patauá (Oenocarpus bataua) na reserva florestal Adolpho Ducke, Manaus, Amazonas, Brasil. Acta Amazónica. VOL. 34(4) 2004: 553 - 558

Vásquez, 2007. Evaluación de Fenología de ungurahui (Oenocarpus bataua Mart.) en el C.E Fitzcarrald, Informe IIAP, Madre de Dios, 30 pp. 